ON THE

\title{
CONSTRUCTION AND USE
}

\section{or \\ A NEW FORM OF CARDIOGRAPH.}

BY

\author{
ALFRED LEWIS GALABIN, M.A., M.D., \\ ASsistant OBSTETRIO PHYsIOIAN TO GUY's HOAPITAI, AND ASSISTANT \\ PHYSIOLAN TO THE HOSPITAL FOR SICK OHILDREN.
}

(Received February 18th-Read May 11 th, 1875.)

THE cardiograph, like the sphygmograph, has retained in the most essential particulars the construction first given to it by Marey, to whom the graphic method owes its chief development, and the alterations introduced by others are improvements of the mechanism in comparatively minor points. The chief feature of the instrument as invented by Marey is that, in order to facilitate the arrangement of the apparatus containing the clockwork, the motion to be recorded is transmitted through a flexible tube containing air. The cardiac motion of the chest-wall is imparted frrst to a cup or hollow disk, called a tambour or tympanum, the rim and back of which are rigid. The interior of the disk communicates by a tube of vulcanized india rubber, not too elastic, with a second tambour, in such a way that the two tambours and the tube enclose an air-tight cavity. The

voL. LVIII. 
front of the second tambour is covered by thin india rubber membrane, and the centre of this is attached to a light recording lever at a point not far from its axis of motion. By this means the motion communicated to the second tambour is magnified, and recorded upon a revolving cylinder called a polygraph. The amount of amplification given to the tracing can be varied at pleasure by adjusting with a screw the position of the recording lever.

Various forms of cardiograph differ from each other chiefly in the construction of the tambour which is applied to the chest over the apex of the heart. Marey first employed a metallic cup closed by a double membrane of india rubber, and having a tube by means of which water could be injected between the two layers of membrane. A convex elastic surface was thus obtained which, when applied to the chest-wall, slightly depressed the region of the heart. The contrivance, however, which he ultimately preferred is a slightly excavated cup, not covered by any membrane, but applied so closely to the skin that the contained air is hermetically enclosed. Within this cup is a spring ending in a pad of ivory, which slightly depresses the skin over the centre of impulse. In this way the motion of the whole surface of the chest contained within the circumference of the cup is communicated directly to the air within it, and thence to the column of air within the flexible tube.

In the use of this cardiograph a difficulty is found in keeping the cup closely enough applied to the skin, and both this and the former are open to the objection that the movement recorded is not that of any one point of the chest, but the mean result of the motion of a considerable surface. Both these disadvantages are avoided in the form of tambour introduced by Dr. Sanderson. The rim and back of this are made of brass, the front of elastic membrane. To the brass back is fixed a flat spring, which is bent twice at right angles in such a way that it overhangs the face of the tambour. Its extremity is perforated by a screw, the point of which rests on the centre of the mem- 
brane, while its head is furnished with an ivory pad. The tambour has also three adjusting screws by which it rests on the wall of the chest. In this way the movement communicated to the elastic membrane and thence to the contained air is that of the very small area immediately in contact with the pad.

It is evident that if in any apparatus for the registration of motion anything of the nature of a spring intervenes between the moving object and the recording lever, the curve recorded will deviate from the true motion whenever any variation occurs in the tension of the spring, and any abrupt movement will be liable to set up oscillation in the instru. ment. The more flexible the spring the greater will be the risk of error. Now, in all the cardiographs hitherto described the recording lever is balanced upon a spring of air, which exceeds all other springs in sensitiveness, and therefore any sudden impulse communicated to the tambour is likely to set up oscillations whose period depends on the joint effect of the motion of the lever and that of the column of air. If, however, the movement to be recorded is suffciently gradual and slow, it may be transmitted without any perceptible modification. This defect in the cardiograph has not escaped criticisur by eminent physiologists, and it may be easily verified by trial that if any abrupt motion be imparted to the elastic membrane oscillations do take place. I cannot do better than quote the words of Professor Rutherford in his lectures on the circulation of the blood, published in the Lancet of November 25th, 1871. "You have seen," he says, "that if you merely touch for a moment the tambour of the cardiograph, you cause the lever of the polygraph in connection with it to describe, not a single wave, but a number of waves. There is first a great wave indicating the touch, and then a number of wavelets of diminishing size. These wavelets are due to the pendulum-like oscillations into which the column of air is thrown."

There is also another mode in which modification may be introduced into the curve. The motion of the heart is really transformed into waves of condensation and rarefaction. 
in the elastic fluid, and these waves are liable to a change in shape as they pass along the flexible tube. It may easily be shown by taking tracings at different points of the arteries of the body, or of elastic tubes containing water, which form a part of a schema representing the circulation, how considerable is the alteration in the form of the pulse wave as it proceeds. In the case of blood or water there is not only a gradual rounding of the curve, but other changes take place from the effects of the momentum of the fluid. For the purpose of the cardiograph, therefore, air is far more suitable than water would be, but the rounding off of sharp points or abrupt motions will occur much more rapidly in a tube containing air than in one containing water, for in the latter case this effect is produced only by the elasticity of the tube, but in the former it depends chiefly on the elasticity of the contained fluid.

In the case of the cardiograph this rounding off of any abrupt features of the curve is probably a positive advantage, for it counteracts, to a great extent, the effect of the other source of error, and the more gradual motion is less likely to call out the oscillations which would be set up if the movements communicated to the lever were as abrupt as the actual movements of the chest-wall.

For the verification of the results obtained by his cardiograph Marey has employed the method of imparting to the apparatus a motion already known, which can be compared with the curve described by the recording lever. For this purpose he made use of an eccentric turned by a handle. The circumference of the eccentric acted directly upon the short arm of a recording lever which was kept in close contact with it by a strong antagonistic spring. The eccentric was cut into such a shape that the curve described by this first lever resembled a cardiographic tracing as obtained by the polygraph. The lever was then attached to the membrane of the first tambour of a polygraph. The motion was thus transmitted by the tube to the second tambour and recording lever, by which a second curve was described upon the revolving cylinder immediately above the 
first. If, then, the instrument were perfect in its indications, the two curves ought to correspond precisely. It was found that the slower the motion of the eccentric the more perfect was the identity between the two, but that if the eccentric were turned more rapidly the effects of the inertia of the instrument became manifest in the second curve. The discrepancy, however, could be made to disappear by increasing the friction of the pen against the paper.

The method of verification thus adopted by Marey is a most valuable and trustworthy one for indicating the limits of possible error. But as applied by him in this instance it proves at most that the cardiac motion, as transformed by a first transmission through the tube, and in which the main ascent and descent are probably already followed by one or more oscillatory repetitions, can be transmitted a second time without any further obvious change. It does not prove that it is possible so to transmit without modification the original motion of the chest-wall, which is shown by other means to have more suddenness than appears in the polygraphic curve. It is evident, indeed, that no very sudden motion could be communicated to the lever by the revolution of an eccentric having a curved margin with which its shorter arm is kept in close contact, even when the handle of the eccentric is turned rapidly.

The sources of possible inaccuracy in Marey's polygraph do not in the slightest degree invalidate the most important result which he obtained by its means, namely, the demonstration of the normal succession of events in the several cavities of the heart and in the aorta. This he obtained by inserting sounds, whose open extremity was covered by an elastic membrane, into the right auricle and also into the right ventricle of a horse, while another elastic ampulla was inserted in the chest-wall over the centre of cardiac impulse, and simultaneous tracings were procured of the three motions. In another experiment one sound was inserted into the aorta and another into the left ventricle. In none of these curves can any suspicion 
attach to the chief points essential for comparison, namely, the main upstroke and main downstroke which denote the beginning and the end of the ventricular systole, and the slight elevation just preceding the ventricular upstroke which indicates the systole of the auricle. Some minor points, however, are seen in the curve which are probably the result of oscillation in the polygraph. This is especially the case with a descending series of waves superposed on the broad systolic summit, immediately after the principal upstroke, which are attributed by Marey himself to an oscillation in the tension of the auriculo-ventricular valves.

The sphygmograph itself, applied directly over the apex of the heart, has been employed both by Marey and also by Professor A. Garrod and other observers for the obtaining of cardiac tracings. The curves obtained in this way, especially those published by Professor Garrod, are much more complex than those described by the polygraph, and show a number of minor points, which appear to be lost when the motion is transmitted through a tube. In attempting, however, to make use of the sphygmograph in this way in cases of heart disease two insuperable difficulties are found. The first is that, when the apex beat is powerful and extends over a wide area, the ivory bars of the instrument touch the chest within the range of the heart's impulse. The curve described represents, therefore, not the motion of any part of the chest, but only the excess of the motion of the spring pad over that of the ivory bars. The result is that some of the features in the curve may often become reversed, and in some cases even the principal upstroke caused by the ventricular systole may be couverted into a downstroke.

The second difficulty arises from the great variation in the extent of the cardiac motion. In the case of a healthy heart the ordinary sphygmograph, which magnifies the motion about ninety times in a vertical direction, may give a curve of moderate amplitude, but when there is much hypertrophy the motion is far too great to be recorded by such an instrument. Even with a magnifying power of 
only twenty the movements of the lever may be so extensive that not only is the curve likely to be modified by the effects of its acquired velocity, but the writing point cannot be retained within the limits of the smoked paper.

The cardiograph represented in the engraving (see p. 360) has been constructed, according to my directions, with the view of obviating these two defects and obtaining the cardiac tracings in all cases directly from the chest. The brass frame of the instrument resembles that of the sphygmograph, except as regards the bar which carries the knife edge, A, through which the motion is transmitted to the long lever. This bar, B, is made up of two parts, of which one slides within the other, and can be fixed by means of a screw, $c$, in whatever position is desired. There is also a second kuife edge, $D$, which can be raised or lowered at pleasure, attached to the same bar at a greater distance from the axis of the long lever. By this means the magnifying power of the instrument, as regards the vertical height of the curve described, can be varied from ten to about a hundred. The brass frame, which in the sphygmograph is rigidly fixed to two parallel bars of ivory by which it is supported, is freely suspended in the cardiograph by means of two transverse rods of steel, E. These are attached by joints, $\mathrm{F}$, which allow both of vertical and horizontal adjustment, to four vertical rods of steel, G, each pair of which is inserted into a bar of wood covered with leather, - by means of these wooden bars the instrument rests upon the chest. They can be separated to a width of nearly five inches, and the instrument can be raised or lowered at pleasure at either end, and in this way it can be adapted to a chest of any size or shape.

In order that vertical adjustments at either end may be possible independently, the brass frame is not in immediate contact with both the transverse bars which support it, but at one end it is suspended by an intermediate piece of brass, I, which, when the instrument is in position, is tightened and made rigid by a screw. The spring which is employed to press upon the centre of impulse is arranged 


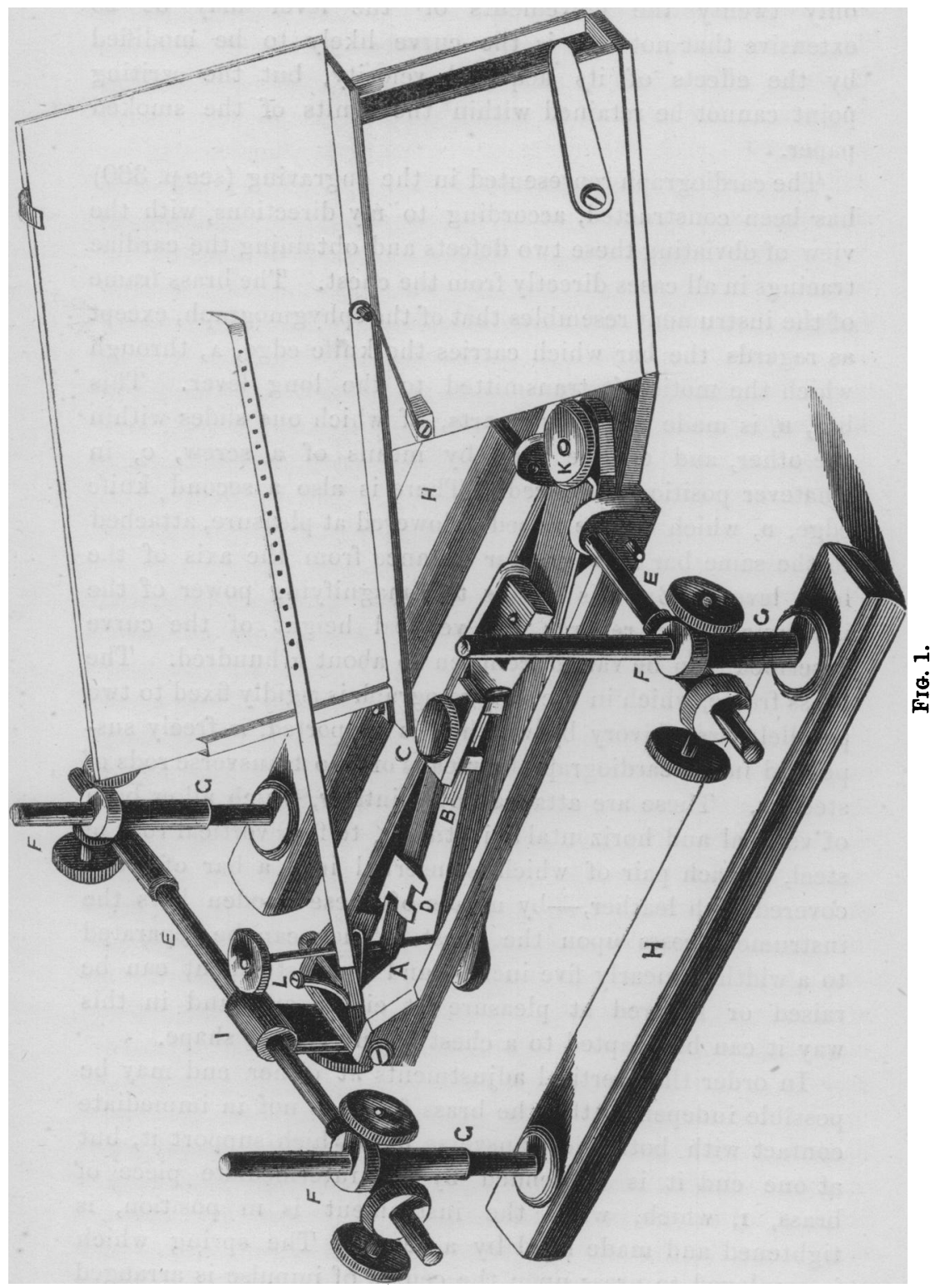


in a mode similar to that adopted in the sphygmograph. The mechanism, however, by which the amount of pressure is finally adjusted is a simpler one than that employed in any one of the various forms of sphygmograph now generally used. This simplification is rendered possible by the fact that, in the case of cardiographic tracings, a knowledge of the exact amount of pressure employed would have little or no significance. The adjustment is effected by means of a screw, $K$, which perforates the short arm of the spring lever, B. The weight of the lever itself is also counterbalanced by a small antagonistic spring.

In this way the pressure upon the point at which the spring pad is applied can be reduced almost to zero, and thus it is easy to obtain with this instrument a tracing representing the backstroke in veins (Fig. 3), which even the weight of the spring of an ordinary sphygmograph is generally sufficient to extinguish.

There are also two small springs, $\mathbf{x}$, of different strength, to depress the long lever and prevent its being jerked away by any sudden motion from the knife edge on which it rests. Either of these can be used or turned aside at pleasure. When the instrument is used in a vertical position it is generally better to dispense with this small spring, since it adds a little to the friction, and it is found that the lever does not become separated from the knife edge, provided that the magnifying power of the cardiograph be so adjusted that its movements have only a very moderate amplitude. If, however, it is desired to take a tracing from a patient in a sitting or standing posture, it is necessary to use the secondary spring, for then the recording lever is no longer kept in position by its own gravity.

The cardiograph may be fixed upon the chest by two narrow straps passed round the body and fastened by buckles. These should be partly elastic, that they may yield a little if the patient makes an inspiratory effort while the clockwork is in motion. In this way the disturbing influence of the muscular movement upon the 
cardiac curve is diminished. As soon, however, as the observer has acquired some dexterity, it will be found sufficient in most instances to hold the instrument against the chest with the hand. In the case of a healthy heart, a uniform and perfect tracing can, as a general rule, be obtained only while the breath is held, and it is best that it should be stopped at the end of expiration. When, however, there is much hypertrophy, the magnifying power required is so much less that the respiratory curve becomes little noticeable in proportion to the cardiac motion, and then the holding of the breath is not so essential. In many cases, when the impulse is very feeble, a more ample curve may be obtained if the patient is placed in a sitting posture, leaning somewhat forward.

In the tracings taken by the direct cardiograph the motions are much more abrupt than in the curves obtained with the ordinary instrument, and the minor elements in the trace are more numerous. This result tends to confirm the conclusion drawn from other grounds, that the transmission through the tube containing air introduces some modification into the motion. With this instrument, moreover, there is greater facility for ascertaining the relation of the chief elevations or depressions of the curve to the impulse or thrill felt by the finger and the sounds or murmurs heard by the stethoscope. For with the tambour cardiograph a slight interval of time is required for the waves to pass along the intervening tube, but in this the movements of the lever follow immediately those of the chest-wall.

It is easy, therefore, for the observer to watch the lever while he rests a finger upon the apex or listens to the heart sounds with a flexible stethoscope, and in this way he may ascertain the significance of the chief features in the curve. Not unfrequently it is found that the thrill which accompanies a murmur is represented in the trace by a vibratile line, and when this is the case a permanent record is obtained of the precise relation of the murmur with respect to the systole and diastole (see Figs. 6 and 11). 
With regard to the possibility that the curve may be modified by the effect of acquired velocity in the instrument, the direct cardiograph, like all other recording instruments, fails to secure absolute fidelity. For the elastic tissues which intervene between the pad of the lever and the heart are in some degree analogous to $a$ spring, and by a variation in the degree of their compression a deviation in the curve might arise. Such variation is likely to be greater, the smaller the pressure employed.

In the case of the heart, since the moving force is greater than that of the radial pulse, a much greater pressure may usually be applied, and, therefore, the liability to oscillation is less than with the sphygmograph, and, $\grave{a}$ fortiori, less than in the tambour cardiograph, in which the lever rests upon a spring of air. As compared with the sphygmograph also, since the amplification in vertical height given to the motion is generally many times less, so many times less also is the effect upon the trace of any given error in the instrument. Another source of error might arise from the long lever becoming separated, in consequence of its acquired velocity, from the knife edge by which it is moved. This is especially likely to occur when the heart's impulse is sudden and strong, and it is a more probable occurrence in the case of the cardiograph than with the sphygmograph, since the motion to be recorded is often much more abrupt. It may be avoided by the use of the small secondary spring, $\mathrm{I}$, which is used to depress the long lever; but in most cases this may be done as effectually, and with less disadvantage, by so adjusting the magnifying power of the cardiograph that the movements of the lever are not too extensive. As a rule, however, it is desirable to compare tracings taken with and without the secondary spring. Since the main upstroke in the curve is usually very sudden, it is probable that the summit which forms the commencement of the systolic eminence often owes something of its height and sharpness to the effect of acquired velocity, but it would seem that, if due precautions be 
taken, the rest of the curve may be relied upon as faithful to the truth.

It does not come within the scope of the present paper to discuss the difficult question of the interpretation of all the elevations seen in the cardiographic tracings, a problem which I have attempted to solve in an article contributed to the 'Guy's Hospital Reports' for 1875, but it may be well briefly to mention some of the most important characters, as seen in the specimens shown in the woodcuts. The systolic part of the trace forms a somewhat square eminence (see Figs. 2, 4, and 10), of which the most important elements are two-first, a sharp-pointed summit at its commencement, probably due to the hardening and rounding of the ventricles; and secondly, a more rounded swelling towards its conclusion, probably caused by the continued pressure against the ribs as the heart is propelled forwards. The former of these is increased in proportion when the heart is dilated, and the latter when it is hypertrophied, and, therefore, by a comparison of the two some estimate may be formed of the ratio which hypertrophy bears to dilatation in any given case.

The diastolic part of the curve, when the heart is healthy, forms a very gradual ascent, commencing at the end of the principal downstroke (Fig. 2). In cases of aortic incompetence a rapid rise takes place, the degree of which indicates the freedom of the regurgitation (Fig. 4). In mitral regurgitation the ascent is also somewhat quicker than normal, in consequence of the rapid repletion of the ventricles caused by high tension in pulmonary and systemic veins.

An important application of the cardiograph is found in the indications which it affords in cases of mitral contraction. In the typical healthy curve (Fig. 2) the auricular systole is expressed by a small elevation just preceding the main upstroke. In some cases of mitral contraction in which a short presystolic murmur is heard, the auricular elevation is increased in height, and is either normal in width or slightly prolonged (Fig. 5) ; in others, in which a 
FIG. 2.-Normal apex tracing.

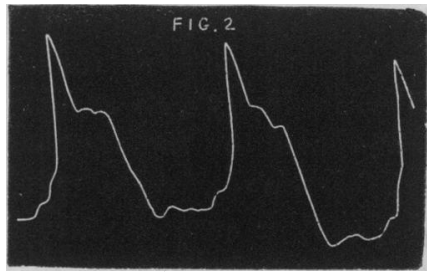

FIG. 3.-Tracing from the subclavian vein in a case of tricuspid regurgitation with mitral contraction.

FIG. 4.-Apex tracing. Free aortic regurgitation.

Fra. 5.-Apex tracing from a case of mitral contraction in which a short presystolic bruit was heard.
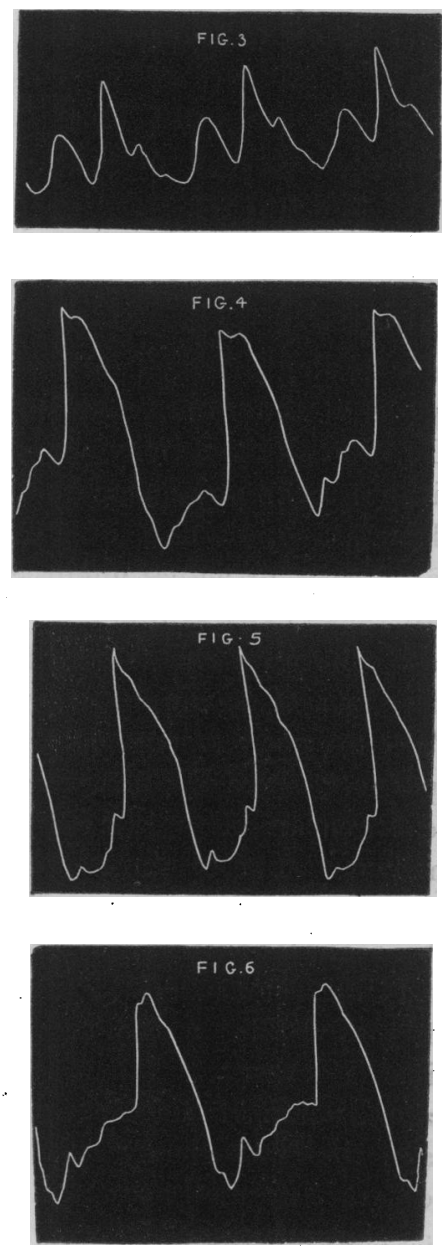

Fra. 6.-Apex tracing. Long and harsh presystolic murmur, accompanied by thrill, running up to the first sound. 
harsh and characteristic presystolic murmur extends over the whole period of rest, it appears to be replaced by a vibratile line which begins to ascend almost from the commencement of the ventricular diastole (Fig. 6). But in another class of cases of mitral contraction, in which there is a soft, or even sometimes a purring or somewhat rumbling murmur, commencing with the ventricular diastole, and either reaching up to the succeeding systole or separated from it by an interval, the character of the curve is altogether different, and appears to point to a different causation for the bruit (Fig. 7). The auricular elevation is either altogether invisible or it appears in its normal position and not prolonged.

There is another important use of the cardiograph in which it serves, not to afford evidence on a theoretical question of the mechanism of a murmur, but as a practical aid to diagnosis. This is found in its application to any point of abnormal pulsation either in the chest or abdomen. In the chest it may be necessary to distinguish between impulse communicated from any one of the cavities of the heart, the pulsation of a tumour, the simple dilatation of an artery, or actual aneurism. The form of the curve in all these cases is different, and in the case of an aneurism some information is afforded by its appearance as to the freedom of communication between the sac and the artery. The ordinary sphygmograph is not usually available for this purpose, since the pulsation is generally too forcible and too diffused to allow a reliable tracing to be obtained by its means. The tracing shown in Fig. 8 was taken from the surface of an aneurism of the arch of the aorta. It will be seen that it is comparable with the sphygmographic tracing of an arterial pulse. The so-called percussion wave is just visible in the ascent, and the dicrotic wave is seen in the descent. When there is no aneurismal sac, but only a dilatation of the aorta, the correspondence is much closer, and the tracing may exactly resemble that of a senile pulse obtained from the radial artery. This result is of interest as showing the fallacy of the view which has been held by 
Fig. 7.-Apex tracing from a case of mitral contraction in which a blowing diastolic murmur, followed by a pause, was heard at the apex only. There was also a short systolic murmur, and a presystolic murmur had been heard previously.

Fra. 8.-From the surface of an aneurism of the arch of the aorta, which formed a pulsating swelling displacing the sternum.

Fig. 9.-From the surface of the liver in a case of tricuspid regurgitation with mitral contraction, taken during respiration.

Fig. 10.-Apex tracing from a case of old mitral disease in which a systolic murmur was heard. After death the mitral orifice was found widely dilated, the left auricle much hypertrophied.

Fig. 11.-Apex tracing from a case of pericarditis in which there was a to-and-fro friction sound accompanied by thrill.
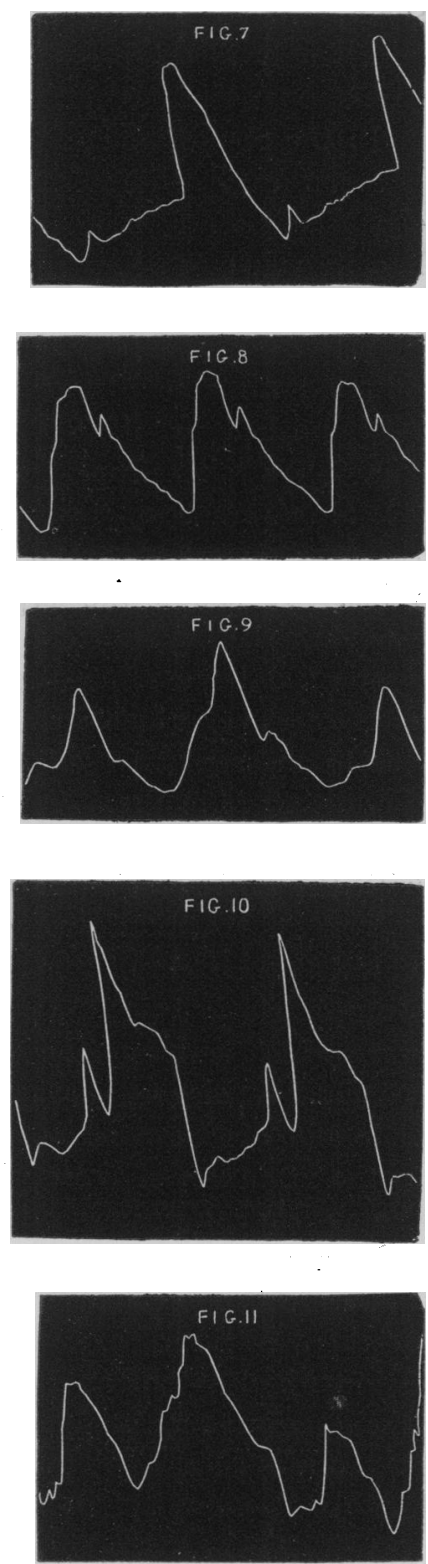
some, that the dicrotism of the pulse is a phenomenon which occurs only in the peripheral arteries, and not in the aorta itself.

In the case of abdominal pulsation the diagnosis may lie between cardiac impulse communicated through the liver, expansive pulsation of the liver due to backstroke in the hepatic veins, temporary dilatation of the abdominal aorta produced by nervous influence, the transmission of pulsation by a tumour, and aneurism. In this case also the transmitted cardiac impulse or the aneurismal pulsation may be distinguished by the shape of the curve. The tracing in Fig. 9 was taken from the surface of the liver in a case of mitral contraction with tricuspid regurgitation, in which there masked pulsation also in the external jugular veins. It shows the typical features of a venous pulse, namely, the anadicrotic wave, preceding the principal summit and caused by the auricular systole, and the katadicrotic wave, which breaks the descent and appears to be due to an oscillation analogous to that which produces the dicrotic wave in the arterial pulse. The last tracing should be compared with that shown in Fig. 3, which was taken with the cardiograph from the subclavian vein of another patient in whom there was tricuspid regurgitation and mitral contraction, the auricular elevation being very strongly marked in the apex tracing. In this case the wave which is due to the auricular systole, and precedes the principal eminence, has a very great proportionate magnitude and distinctness, and this curve may be commended to the attention of those who find it difficult to believe that the auricle can, in any case, acquire force sufficient to produce a murmur by its contraction. In the descending curve are two secondary waves which seem to correspond to the dicrotic wave, and the tricrotic wave or second oscillation which is sometimes also seen in the arterial pulse tracing when tension is low. 